\title{
The Impact of Student Location in a Global Design Project
}

\author{
Keilah Bias, Daniel Larsen, Libby Osgood \\ Dalhousie University, University of New Brunswick and University of Prince Edward Island \\ Email: [keilah.bias@dal.ca,dlarsen@unb.ca,eosgood@upei.ca]
}

\begin{abstract}
Various engineering programs in North America enable students to immerse in international projects. Some students travel with NGOs and perform engineering work as part of their curriculum, and some design projects for hypothetical clients in other parts of the world. The authors designed a charcoal press for Kenyan farmers as part of their 2 nd year design class. Due to the success of the design, the project was brought to Kenya, and was modified to be built to the materials and equipment that were available in the rural communities. One of the authors travelled to deliver the design, participated in building the device, as well as trained the clients to perform the process. The second author stayed in Canada.

The authors present their experience on this two-stage design process, where the students were exposed in both designing from a distance and being immersed in the international setting of the project. Writing from students' perspective, different points on how the project was effective on engraving design principles to students were shared. Students also reflected on their experience and developed recommendations on how the experience can be improved for future students who will take similar programs.
\end{abstract}

Keywords: Experiential Learning, International Design Project, Student Reflection, Student Perspective, Design, Heuristics

\section{INTRODUCTION}

The increased globalization of today's world presents greater challenges to the global engineer. ${ }^{[1]}$ The "Flattened world" presents more difficult problems, more complex systems, more intricate ethical conflicts, and greater influences from human behavior and sociology. Ethics itself is a challenging concept to transfer to engineering students. Expanding this to global ethics in international design is even a more abstract concept to teach separate from experience. ${ }^{[2][3][4]}$

While the essence of engineering is the design process and problem solving, it is essential to develop skills to adapt into different contexts. Cross-cultural immersions and international design projects aim to train engineering students in this area. This is done in multiple ways through encouraging students to travel and work with an international NGO by giving them credit hours for their work, by creating international design projects as part of the capstone projects, or simply incorporating multicultural or international case studies in class work. Some engineering organizations, such as Engineers Without Borders, also expose students to opportunities to work on projects for developing countries from their own campus.

According to recent studies, international projects expose students to multi-location developments that are prevalent in today's outsourcing market, exposes students to the importance of diversity in developing better designs, as well as students learning about other cultures. ${ }^{[5]}$ Previous research found that travel also enhances learning outcomes. Students who went to the place where the problem occurred presented better teamwork and awareness compared to those who designed from a distance. ${ }^{[6]}$ Even beyond graduation, students tend to retain lessons learned and behave more ethical when exposed to community-based and multi-cultural projects. Students recall that the experience enables them to balance social, economic and environmental components of design. Particularly, the students commend their increased interest in the social component of their design, including ethics. ${ }^{[7]}$ Diversity also teaches engineering students that different paradigms or perspectives may present better ideas than theirs. This improves teamwork, open-mindedness and lifelong learning skills. ${ }^{[8][9]}$

Both the Canadian Engineering Accreditation Board (CEAB) and the Accreditation Board for Engineering and Technology (ABET) emphasizes design abilities in context of multi-faceted attributes. International and community based projects present this holistic development advantage to students. As long as enough time for reflection is provide, benefits for students are substantial.

This paper presents the experience and reflection from a student experience on a two-phased international design project. The design was initially developed as part of a $2^{\text {nd }}$ year design project. It was developed for Mikinduri Children of Hope (MCOH), a non-profit organization that does work in rural communities in eastern Kenyan. The initial problem presented was the prevalent scarcity of fuel sources in Mikinduri, Kenya. The locals previously turned to cutting wood from forests for cooking and heating. This concerns environmental groups for potential deforestation. As a result, the residents are now producing charcoal from agricultural waste to substitute as a fuel source. However, the current process of doing so involves using a steel plunger and produce one charcoal briquette at a time. It does not produce enough yield to justify the time, tediousness and poor ergonomic nature of production. $\mathrm{MCOH}$ presented this project in a desire to improve the process and provide potential sources of income to the Kenyan villagers. 
The initial design and prototype was developed at the University of Prince Edward Island (UPEI) in the winter of 2014. Feedback from the Kenyan clients was received electronically, and the students only had one opportunity to send a prototype due to long distance. A paper presenting the reflections on the first phase of the design project was previously published 2015. ${ }^{[10]}$ Since then, the final design was brought to Kenya through an $\mathrm{MCOH}$ mission trip in 2015. One of the authors had the opportunity to travel to Kenya and implement the design in the actual community where it was intended for use.

In this paper, the students present their comparison and reflection of the experiences from performing the design process from two different locations. The authors also recommend different ways on how educators can incorporate some concepts learned in the international project in design classes for the benefit of student learning.

\section{PHASE 1: DESIGNING FROM A DISTANCE}

The first stage of the project involved designing a charcoal press for use in rural Kenya in partnership with the $\mathrm{MCOH}$ foundation. The project spanned 2 semesters as a part of a second year design courses. Multiple designs were first developed until the first initial detailed design was done.

\subsection{Initial Design}

Charcoal can be produced by burning organic agricultural waste in a barrel and then sealing it to produce a low oxygen environment. This will drive off the water and impurities leaving only the carbon structure. The charcoal can then be extracted from the barrel and crushed to produce charcoal powder. The charcoal powder is then mixed with cassava and water to form a paste. The paste is pressed and left to dry. The resulting briquettes can be used for cooking and heating and produce almost no smoke when burnt.

The first successful design was the cube press, which is shown in Figure 1. The press was designed to compress the charcoal paste into a mold. The mold was placed on the far side of the box and the charcoal paste was compressed by a flat plate pushed by a screw mechanism. The compactness of this solution was seen to be ideal and early testing of the prototype in the lab proved to be very successful.

\subsection{Final Design}

Designing from a distance was challenging because it is difficult to fully understand how the end user will use the device. A prototype of the cube press was sent to Kenya with the MCOH team. A video of the presses first use was recorded and sent back to the authors for review. This resulted in an initial solution that worked fine at the university, but was not ideal for use in Kenya. The initial press was designed to operate on a table. This was never a problem as there were always tables in the lab. In Kenya the device was used outside where no table surfaces were available. Using the initial prototype on the ground made it much more difficult to operate. The problems with the cube press prompted a complete redesign.

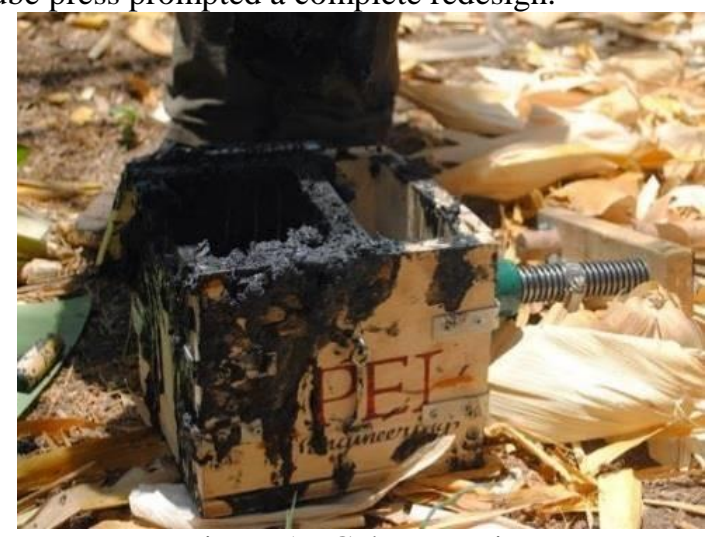

Figure 1 - Cube Press in Kenya

The second prototype, shown in Figure 2, was designed to address the weaknesses of the cube press which did not adequately compress the charcoal, and took too long to operate due to the threaded screw. The second prototype can press sixteen liters of charcoal with each downward movement of the handle. The prototype was fabricated at a welding shop near the university.

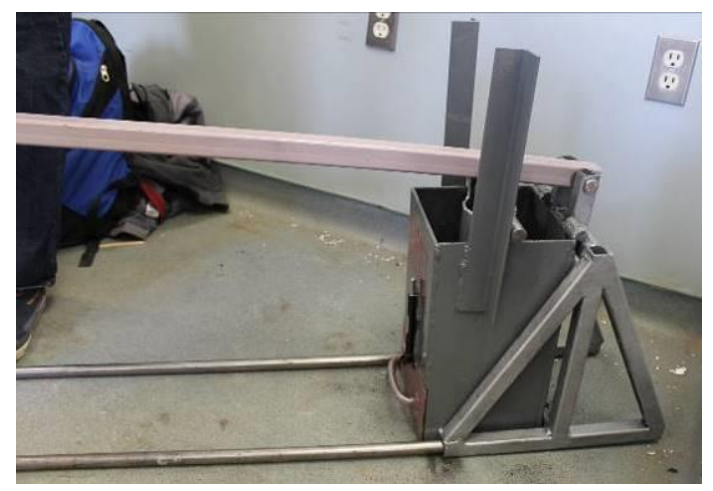

Figure 2 - Final Prototype at UPEI 


\section{PHASE 2: ITERATING AND IMPLEMENTING THE DESIGN IN AN INTERNATIONAL SETTING}

After the design course was finished, the students decided to continue developing the device outside of class for potential implementation. The second phases involved improving the design to be more adaptable for implementation to different possible countries, designing simplified user instructions, and refining the design.

A year later, the drawing package and user instruction kit was brought to Mikinduri, Kenya. It was during the MCOH trip in February 2015. The team was comprised of nurses, a doctor, UPEI engineering students and a professor. The trip also involved volunteering in schools, hospitals and households.

\subsection{Building the Local Prototype}

Building the prototype was performed by partnering with a local "polytechnic", which is Kenya's term for a community college. The polytechnic's chair was keen on building the design for their community, and having their welding and carpentry students perform the work to create the device.

The college only had a hand held electric drill, an old welding machine, a hack saw and a grinder. This made constructing the device significantly slower and harder. The school also does not practice, nor promote, Canadian standards for safety practices. Sourcing materials was also challenging because of the rural location, distance to sources, and availability of required materials.

Due to the limitation of the facilities and equipment, there were numerous modifications done to the design as it was built. However, the device was designed to be adaptable to these modifications, because they were expected to occur. It was finding an alternative way to cut, drill, and weld that presented the greatest challenges during construction.

\subsection{Testing and Implementation}

After the design was built, testing the device was performed in front of a diverse crowd from the community. There were some politicians, NGOs, farmers, students and local villagers. They were all present to witness the entire process from preparation to drying the charcoal.

While performing the charcoal making process, not all of the safety precautions that were listed in the project's original test plan were followed. Their culture has different safety practices, which may be considered unsafe in North American standards. They were touching the burning barrel with their hand, women were holding knives while breaking large corn stalks with their knees. This seemed to be a norm and acceptable. This did not affect the quality of the test that was done, but it changed the order of the steps in the plan, and eliminated some steps completely.

There was an abundance of stalks and agricultural waste, as well as cassava. The simplifications in the process were also found effective. The charcoal briquettes that were pressed were high quality. The drying time was shortened to less than 24 hours compared to a week when it was performed in Canada.

The most major problem while pressing charcoal was the handle bending due to its poor quality. It was modified later on with a bigger handle to prevent further bending. This was a major modification to the design as the handle is a key feature of the device. The larger handle is shown in Figure 3. Other complications in pressing caused by the imperfections in building the press was also observed.

The second phase of the testing involved demonstrating how the charcoal burns as well as regular charcoal. This test was successful.

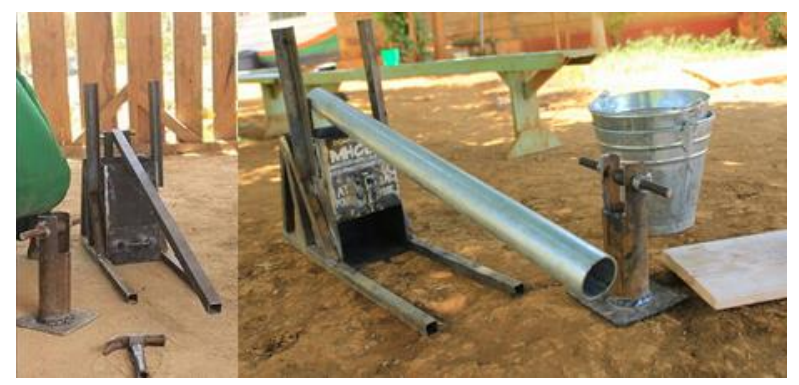

Figure 3 - Handle Modification to the Press

Overall, the implementation was successful. The villagers were very engaged, interested and attentive to instructions. They were also working together to perform the work and learn the process to apply in their community. Having a connection through the local NGO was important to have a connection to the community and for the clients to buy in. 


\section{IMPLICATIONS}

\subsection{Design Process}

Having an international client made it difficult, from a distance, to assess their real needs and wants. Though $\mathrm{MCOH}$ gave as much information as possible, it was hard to comprehend the situation from second-hand perception. None of the designers have been to Kenya or Africa. Designers tend to think of ideas based on the context of what's familiar, even with extensive research performed.

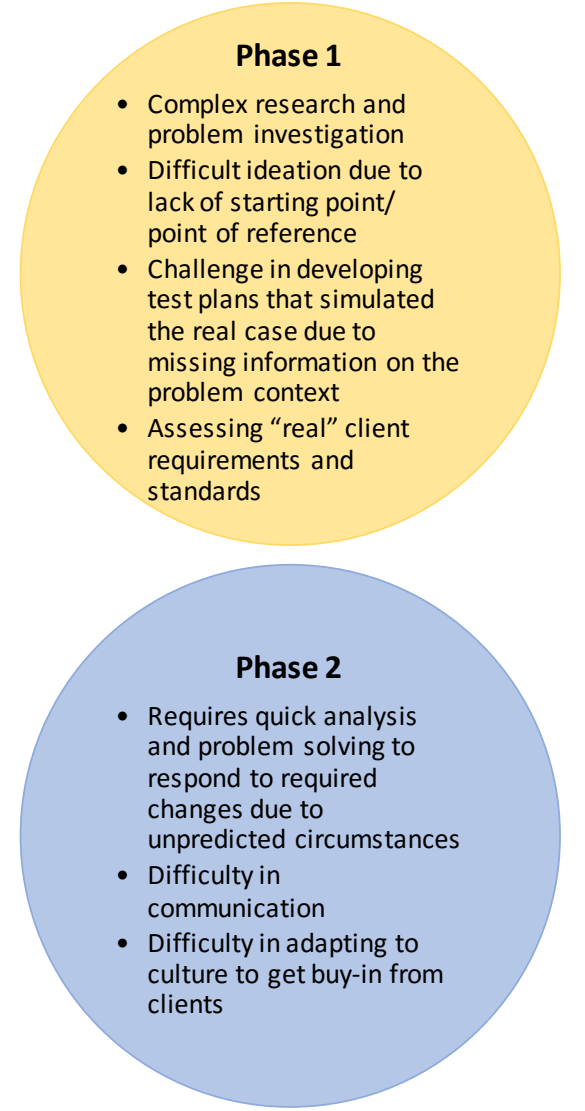

Figure 4 - Main challenges during each phase

Being unable to personally experience the environment and client culture makes it hard to evaluate the design's feasibility during ideation. It was also difficult to create build and test plans. There were numerous assumptions. Even with the proven effectiveness of the design in terms of technical requirements, there was no means to determine the social acceptability of the change from the clients' perspective. After coming to Kenya, it was realized that most of these assumptions are very difficult to infer without personal investigation. The build and test plans had to be redone to adapt to the clients' culture. The students learned to make these plans as general as possible and let the users decide on some details by their discretion. This is a special case as most technical instructions are created to be detailed to reduce variation. The students are only able to learn this through iterative work and experience with the project.

The main challenges in the second phase was responsiveness to changes. This was dealt with by having adequate analyses. A risk assessment would have also been appropriate.

\subsection{Overall Experience}

Critical thinking and creativity were both challenged during the first phase of the project. Not having a starting or reference point to the problem required intensive research. As a student, this was challenging. It required a significant portion of the time due to the number of iterations and amount of brainstorming required. It was certainly a more perplexing project. It provided enough challenge to motivate the students to work hard, learn more, and expand their perspective. Results were apparent as the design won multiple recognitions since it has been finished in 2014, and after its iteration. The students also received one of the highest marks in class.

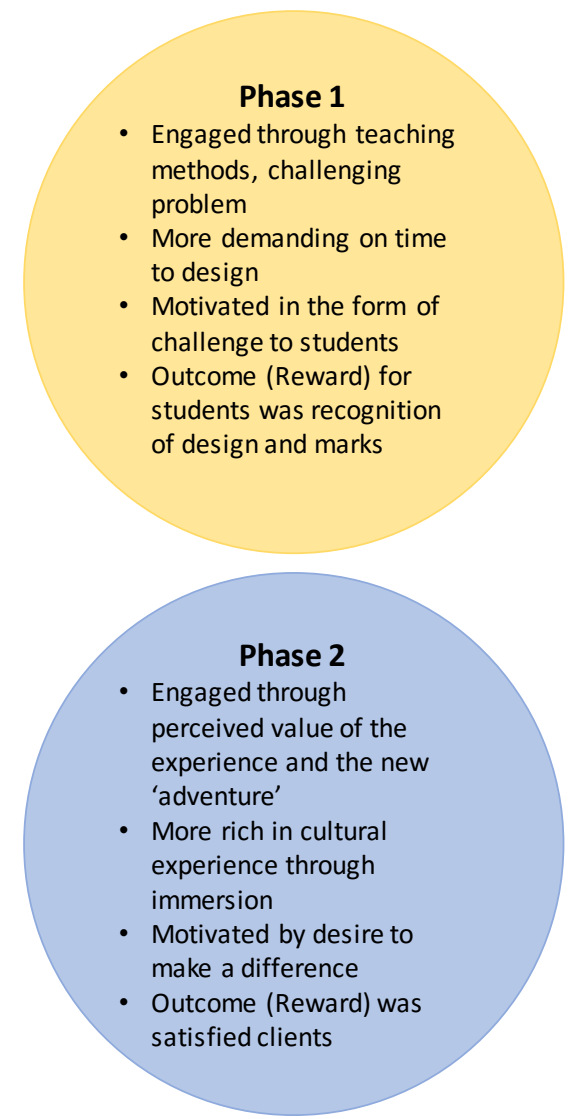

Figure 5 - Student Experience Highlights 
The cultural experience in the second phase of the project was rich. Communication was a significant challenge as translators were required the majority of the time because the locals speak very little English. Having clear drawings and a plan lessens the conflicts that could have happened. Furthermore, the author was the only female in the team while building the prototype in Kenya, and was the one directing the project to completion. The students were surprised to find that she is completing an engineering degree, and remarked that it was only a male profession in Kenya.

Because of the project's success, the design was brought into an international student competition as well as published in local and international articles. This expanded the students' experiences expanded from designing and class to attending networking events, international competitions, conferences and interviews.

Because of an enriched experience, the student engagement was more evident. This was key to the learning outcomes of the project. The authors, as students believe that these learning outcomes are a function of both the type of problem, as well as the teaching methods.

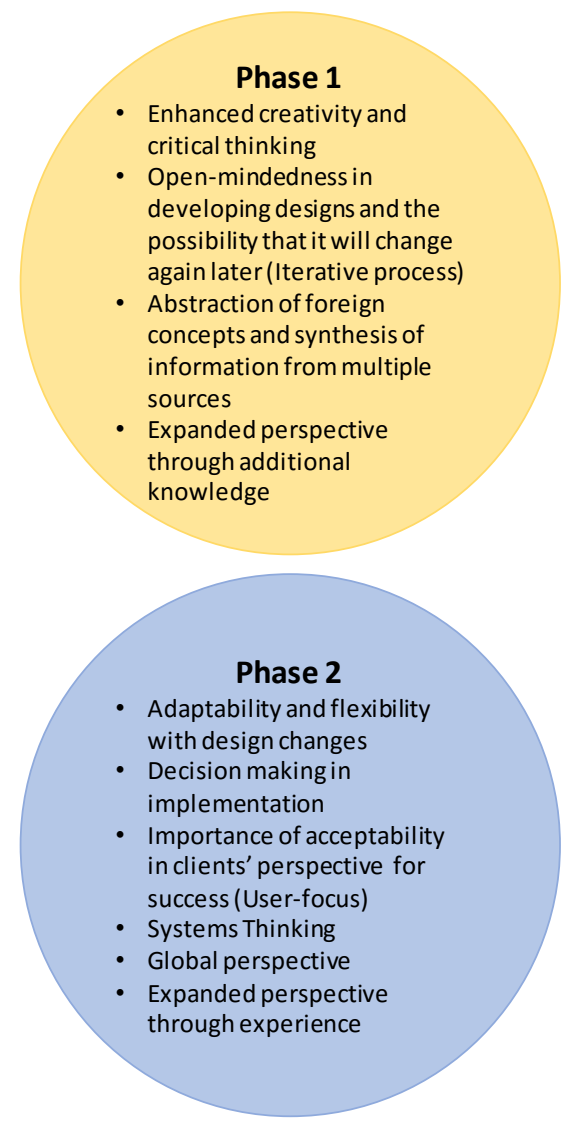

Figure 6 - Key learning outcomes from students' perspectives

\subsection{Learning Outcomes}

The quality of learning was not found to have been compromised by designing the project from a distance and instead some aspects were enhanced. Through effective teaching methods and course design, the CEAB graduate attributes were all successfully incorporated in both phases of the projects. However, the attributes that were emphasized differed for each phase are summarized in Figure 6.

Ultimately, the projects' design also resulted to a successful solution to the problem even if it was designed from a distance. This was from a combination of factors that will be discussed in the next section.

\section{SUCCESS FACTORS FROM STUDENTS' PERSPECTIVE}

The following graphic summarizes the success factors from the students' perspective. Being written within the context of education, the main driver of the dynamics of learning is within the professors' influence. The outcomes, however, can be defined from the context of learning and being able to produce effective and sustainable solutions. Reflection and application of knowledge are two factors that students have more influence (whether or not they are aware of).

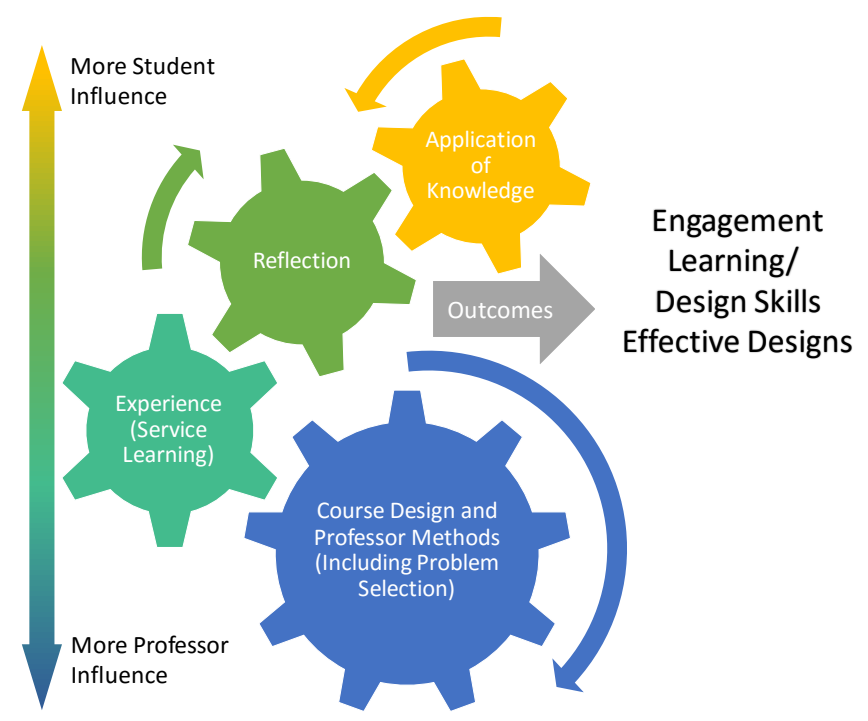

Figure 7 - Success Factors 


\subsection{Course Design and Professor Methods}

The course design gave students full ownership of their problem. It was also made clear during the course and classes that professors expected students to evidence their learning as applied to their projects. Marks were allotted to work that evidence of a quality design process, and less technical components such as teamwork, iterations, professionalism and the like. Selected projects were also mostly community based, or has that component. This allowed the instructions to have a common theme directed towards user-focus and sustainability.

Professors also made an effort to make the clients closer to the students. One of the professors travelled to Kenya multiple times in the past, and provided as much information as possible. Questions to challenge the design were always present during progress reports to direct the students towards the right direction.

\subsection{Service Learning}

Compared to industry-type projects, the motivation with community-based project is much different. Improving the livelihood of another community in need rather than optimizing a product to make better profit holds a greater moral weight and drives greater conviction in terms of the student's desire to spend time, even outside of class, solving the problem. Though the designers were not able to communicate directly to the clients during the first phase, seeing the potential to make a difference is a significant factor in the success of the design. Distance and communication were hurdles, but overcoming them presented a different skill in research, information synthesis, and critical thinking.

Incorporating community-based projects focuses students in designing effective, user-based solutions. Experiential learning has been an acceptable component to engineering education that strengthens competitiveness and skills in students. However, service-learning and community-based projects envelop the expertise with an awareness of engineering's societal and environmental responsibility. Most engineering practices in the profession are driven by economic agenda, or monetary benefits. If the core foundation of the purpose of engineering, which is, 'to make better use of the world's resources while bettering human life' was emphasized, better designs may be developed while still gaining returns on investment. These types of projects also shift students' perspective on prioritizing constraints and focus in projects to ensuring quality is defined by the user requirements and needs. ${ }^{[11]}$ Community-based projects and service learning improves empathy, and engraves the importance of valuing the greater good as a motivation for successful design. It also promotes a positive impression on the students, as the outcomes of the projects are for the betterment of the society, and contributes in a constructive way.

\subsection{Perspective Expansion}

A critical component that contributed to the success of the project was the expansion of the author's global perspective. Working with the $\mathrm{MCOH}$ foundation allowed for a better understanding the Kenyan user. Receiving feedback from the users on the first prototype revealed that the original solution did not adequately consider the differences between using the press in the universities lab and using it outdoors in Kenya. It is common with internationally themed design projects that the solutions are designed from the student's local perspective (phase 1). Working with international clients forced the authors to think about the problem from the user's perspective (phase 2). The influence of a global perspective is illustrated in Figure 8.
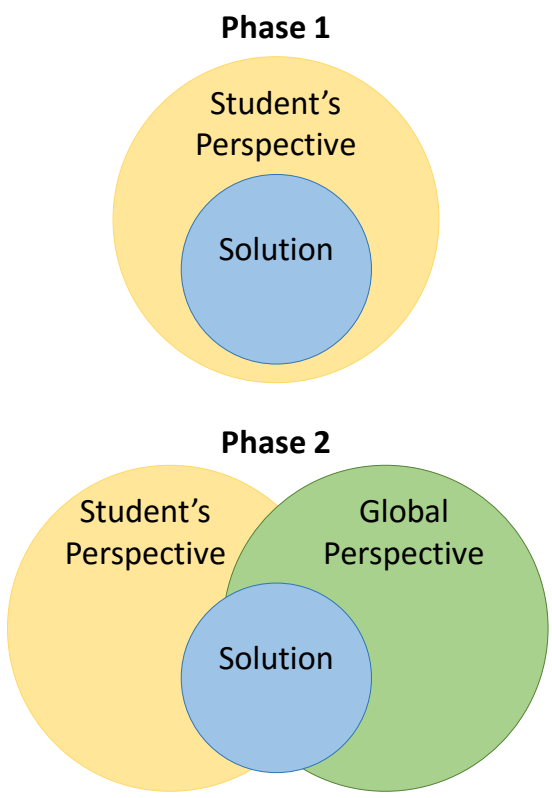

Figure 8 - Perspective Expansion

\subsection{Adequate Reflection}

Reflection was a critical to the success and quality of the student experience in terms of engineering education and design. Without the reflection on these experiences, which are documented on the two papers published on the experience, there could have been less long term meaning from the project for the students. The introspect provided through the exercise allowed the authors to identify key concepts that are transferrable to any project.

There can be multiple benefits and personal development that could be gained from the experience, but the ultimate goal was to teach design. It might be a more difficult benefit to quantify, but reflection reinforces the learning by connecting student experience to technical concepts. It involves intellect and emotion, and may be time consuming, intense and complex. However, it engages the cognition in the experience much tighter. ${ }^{[12]}$ It is a 
catalyst to extract the most meaningful parts of the experience, why it was key to their engineering practice, and what life-long lessons were learned. Reflection helps students realize differences between getting the right solution versus merely getting an answer. [13] [14]

Reflection also enforces self-awareness, leveraging students' strengths and being more considerate of the bigger picture. It supports the technical tasks by ensuring that it is paired with maturity in multiple soft skills and an ethical practice. It motivates students to continually improve their design skills because it shows personal progress. Ethics is also strengthened in the process. Telling one's self an account of what occurred exposes character and behavior that may have not been apparent to the individual at is has been transpiring, and leads to selfevaluation. Overall, reflection builds character for an overall better professional and engineer.

Reflection is performed in multiple contexts, but has also been proven effective in engineering education. It is recommended that reflection be incorporated more into experiential learning, especially community-based and international projects to optimize student experience.

\section{RECOMMENDATIONS}

The students recommend that professors instill more service learning components to improve design abilities of students. This does not have to be in the form of an international project, if it is not feasible. Community-based projects can be performed in closer NGOs or neighborhood. Having the experience to design something with a greater morale and social reward than monetary value at least once in their education or career can make a significant difference in the design ability of students.

If having close clients is not possible, finding ways to make the client closer can be done in other ways. Watching documentaries or reading multiple literature about the problem or the situation can help patch the missing information about the context. This can significantly improve students' perception and remove their bias on their developed solutions.

Giving more connection between engineering and the humanities, including social sciences, is also important. It is difficult to teach user-focus or cultural implications to design. Setting up ways to make students realize the importance of the human component to their solutions enables them to be more effective designers. Experiencing this through service learning can teach this concept very well. Professors can also incorporate this in their courses by asking students to define how they expect their clients to receive and perceive their design. Designing a user experience or writing requirements through use cases or user stories can also be done.

Finally, more meaningful student experiences and more conducive learning can be designed by making more room for reflection. Oftentimes students learn one concept, study for the exams, and then forget everything after that. This is mostly because students are focused more on getting the marks and conversely miss out on the learning. While reflection has numerous benefits as discussed earlier, it does two things from a student's perspective: (1) Students perceive that their instructor cares about what they learn. A professor asking a student to recall their experience and talk about their learning process and then later on reading it gives the impression that the professor is curious what the students experienced. This can be a motivational factor for learning. (2) Reflection alleviates the 'heaviness' from the technical theory. It gives students a break from the intensity of the concepts that they are learning. They get a reminder that they are present to learn.

Reflection and introspect is not one's "natural" way of thinking. It usually involves thinking about someone's thinking process. To do this in the context of problem solving means being able to detect success factors and areas for improvement. Conversely, it means being a better designer every day. ${ }^{[15]}$

\section{CONCLUSION}

This paper serves as a part of a reflective exercise on this project. The authors were able to account for their experience in designing the device, and how it has helped them become better students, designers and engineers. Concepts that were learned from this project were transferrable to approach any problem or project. It has helped in gaining an additional perspective, to view any problem from the perspective of the intended user.

The two student authors were from extremely different backgrounds. One came from an immigrant family from a developing country, while another grew up on a Canadian farm. Both brought two different perspectives in the project and the reflection. Both also learned some shared and different experiences. The significance of user-focus and reflection in design are concepts that students hear, but are very hard to comprehend. The authors were left with the lesson of the importance of making the users "close" and adapting their perspective, and different ways to do so.

The authors recommend that educators give students an opportunity to immerse in service learning through community-based projects. The impact of these are substantial in teaching ethics, social awareness, and lifelong learning. 


\section{REFERENCES}

${ }^{1}$ National Academy of Engineering. Educating the Engineer of 2020: Adapting Engineering Education to the New Century. Washington, DC: The National Academies Press, 2005. doi:10.17226/11338.

${ }^{2}$ Del Vitto, C. (2008). "Cross-Cultural "Soft Skills" and the Global Engineer: Corporate Best Practices and Trainer Methodologies. Online Journal for Global Engineering Education Vol. 3 Iss. 1, 1-9.

${ }^{3}$ Jordan, W. (2009, June), Ethical Issues Related to International Development Projects Paper presented at 2009 Annual Conference \& Exposition, Austin, Texas. https://peer.asee.org/5483

${ }^{4}$ Carroll, M., \& Barakat, N. (2005, June), Globalization of Engineering Ethics Education Paper presented at 2005 Annual Conference, Portland, Oregon. https://peer.asee.org/14952

${ }^{5}$ Ellingson, J. L., \& Greene, C. S., \& Morgan, S. E., \& Silvestre, M. A. R. (2012, June), An International Multiyear Multidisciplinary Capstone Design Project Paper presented at 2012 ASEE Annual Conference, San Antonio, Texas. https://peer.asee.org/2093

${ }^{6}$ Layer, J., \& Gwaltney, C. (2009, June), International Capstone Design Projects: Evaluating Student Learning And Motivation Associated With International Humanitarian Projects Paper presented at 2009 Annual Conference \& Exposition, Austin, Texas. https://peer.asee.org/5067

${ }^{7}$ Wright, H., \& Phillips, L., \& Mihelcic, J. (2008, June), International Senior Design: Assessing The Impact On Engineering Students After Graduation Paper presented at 2008 Annual Conference \& Exposition, Pittsburgh, Pennsylvania. https://peer.asee.org/3367

${ }^{8}$ Evers, C. T. (2011, June), Views of Diverse Groups of International and American Students Concerning Business, Cultural, and Ethical Issues Paper presented at 2011 Annual Conference \& Exposition, Vancouver, BC. https://peer.asee.org/18728

${ }^{9}$ Edington, S., \& Eljamal, M., \& Pang, S. (2005, June), Gaining International Competence: A Multi Faceted
Approach To International Engineering Education Paper presented at 2005 Annual Conference, Portland, Oregon. https://peer.asee.org/14886

${ }^{10}$ Bias, K. M. T., \& Larsen, D., \& Osgood, L., \& Gamble, A., \& Montgomery, S. P. (2015, June), Students' Perspective on the Effectiveness of Design-Based Curriculum during an International Design Project Paper presented at 2015 ASEE Annual Conference and Exposition, Seattle, Washington. 10.18260/p.24773

${ }^{11}$ Catalano, G. (2007, June), Engineering In A Morally Deep World: Applications And Reflections Paper presented at 2007 Annual Conference \& Exposition, Honolulu, Hawaii. https://peer.asee.org/1574

${ }^{12}$ Rodgers, C (2002). "Defining Reflection: Another Look at John Dewey and Reflective Thinking". Teachers College Record, 104 (4), 842-866.

${ }^{13}$ Siewiorek, N., \& Shuman, L., \& Besterfield-Sacre, M., \& Santelli, K. (2010, June), Engineering, Reflection And Life Long Learning Paper presented at 2010 Annual Conference \& Exposition, Louisville, Kentucky. https://peer.asee.org/16615

${ }^{14}$ Use of a reflection journal in a third year engineering project course A.L. Steele Dept. of Electronics, Carleton University asteele @ doe.carleton.ca ${ }^{15}$ Jäkel, F., \& Schreiber, C. (2013). Introspection in Problem Solving. The Journal of Problem Solving, 6(1). doi:10.7771/1932-6246.1131

Proc. 2013 Canadian Engineering Education Association (CEEA13) Conf CEEA13; Paper 84 Montreal, QC; June 17-20, 2013

Engineering Accreditation Commission. (2012, October 27). EAC Criteria. Retrieved from ABET: http://www.abet.org/wp-content/uploads/2015/04/eaccriteria-2013-2014.pdf

Hiles, D. (2001). Heuristic Inquiry and Transpersonal Research. London, UK: Centre for Counseling and Psychotherapy Education. 